livraisons

d'Histoire

de l'Architecture

\section{Livraisons de l'histoire de l'architecture}

$38 \mid 2019$

Varia III

\title{
La cathédrale transfigurée : Notre-Dame de Paris dans les images de la fin du Moyen-Âge ( $\mathrm{XV}^{\mathrm{e}_{-}}$ $\mathrm{XVI}^{\mathrm{e}}$ siècle)
}

The transfigurated cathedral: Notre-Dame de Paris in the pictures of the end of the Middle Ages (XVth-XVI ${ }^{\text {th }}$ centuries)

Die verklärte Kathedrale: Notre-Dame de Paris in spätmittelalterlichen

Darstellungen (15.-16. Jahrhundert)

\section{Raphaële Skupien}

\section{OpenEdition}

Journals

Édition électronique

URL : http://journals.openedition.org//ha/1462

DOI : 10.4000/lha. 1462

ISSN : 1960-5994

Éditeur

Association Livraisons d'histoire de l'architecture - LHA

Édition imprimée

Date de publication : 15 décembre 2019

Pagination : 23-37

ISSN : 1627-4970

Référence électronique

Raphaële Skupien, «La cathédrale transfigurée : Notre-Dame de Paris dans les images de la fin du Moyen-Âge (XVe-XVIe siècle) », Livraisons de l'histoire de l'architecture [En ligne], 38 | 2019, mis en ligne le 02 janvier 2021, consulté le 04 février 2021. URL : http://journals.openedition.org//ha/1462 ; DOI : https://doi.org/10.4000/lha.1462 
Par Raphäle SkUPIEN

\section{LA CATHÉDRALE TRANSFIGURÉE : NOTRE-DAME DE PARIS DANS LES IMAGES DE LA FIN DU MOYEN ÂGE (XIV ${ }^{\mathrm{e}} \mathrm{XVI}^{\mathrm{e}}$ SIÈCLES) ${ }^{1}$}

L'incendie du 15 avril 2019 a réaffirmé le statut de monument emblématique de la Ville de Paris, et de la France, dont jouit Notre-Dame de Paris aujourd'hui. Sur les portants de cartes postales, la cathédrale peut être colorée en vert, en rose, ornée de motifs psychédéliques, stylisée, sortie de son contexte urbain... ses deux tours ne trompent personne! Cependant, l'archevêque de Paris, l'archéologue du bâti et le touriste nippon ne portent pas sur le monument le même regard. Il en allait de même au Moyen Âge entre le roi et le bourgeois, entre locaux et étrangers, etc.

Il ne sera pas utile de revenir ici sur l'histoire de Notre-Dame ni sur son inscription dans l'espace parisien depuis la fondation d'un groupe épiscopal paléochrétien jusqu'à la reconstruction du complexe cathédral aux XII ${ }^{e}$-XIII ${ }^{e}$ siècles puis aux modifications intervenues à l'époque moderne ${ }^{2}$. La vision que nous avons aujourd'hui du monument est largement tributaire de ces dernières puisqu'elle fut blanchie à l'extérieur comme à l'intérieur (effacement de la polychromie, remplacement des vitraux colorés par les verrières blanches des frères Le Vieil au milieu du XVIII ${ }^{e}$ siècle), que son portail fut élargi par Soufflot en 1771 et qu'une partie de son décor sculpté fut déposé à la Révolution. Les restaurations entreprises par Lassus et Viollet-le-Duc à partir de 1843 ont abouti à une restitution partielle voire une recréation du décor intérieur, des oculi, des sculptures extérieures et de la flèche mais non de la polychromie du frontispice pourtant connue.

Jusqu'au XVII siècle, la cathédrale parisienne est suffragante de Sens. L'importance qui lui est accordée semble alors intimement liée au statut de la Ville de Paris, capitale du royaume de France : au cœur de Paris, entre les deux bras de la Seine qui l'isolent de la Ville (rive droite) et de l'Université (rive gauche), le siège de l'évêché occupe la partie orientale de l'île où il s'inscrit en contrepoint du siège des institutions royales, le Palais de la Cité rebâti sous Philippe le Bel. Fortement polarisée, l'île de la Cité concentrerait à l'ouest, la dimension temporelle du pouvoir monarchique, à l'est, sa dimension sacrée, manifeste à travers les rituels décrits

1. Cet article est issu d'une conférence donnée à deux voix, le 16 janvier 2019 pour la Société des Amis de Notre-Dame de Paris, avec Judith Förstel qui signe la première partie.

2. Voir par ex. : Monographie de Notre-Dame de Paris présentée par Jean-Michel Leniaud, Paris, éd. Molière, 2008 (fac-sim.). Notre-Dame de Paris, Paris, 2012 (coll. "La Grâce d'une cathédrale "); les fiches thématiques publiée par les scientifiques au service de la restauration de Notre-Dame de Paris après l'incendie du 15 avril 2019 (sur le site de l'association : https ://www.scientifiquesnotredame.org/). 
dans les textes. Or, si l'histoire factuelle de Notre-Dame de Paris est bien connue, une histoire sensible reste à écrire. Sans prétendre combler ce vide, cette étude des plus anciennes représentations de la cathédrale permet de mieux saisir les motivations qui ont poussé les hommes du Moyen Âge à choisir la cathédrale plutôt qu'un autre monument pour "incarner Paris » ${ }^{3}$. Est-on seulement bien certain que le monument figuré par les peintres ait été identifiable par leurs contemporains? Les sources iconographiques - mises en regard des sources textuelles, littéraires ou documentaires - et des données archéologiques, éclairent sur la perception qu'en ont eue les contemporains, en fonction de leur origine sociale ou géographique. Si l'examen des modes de représentation révèle des sensibilités différentes à l'égard du monument, il met en évidence l'évolution de son statut et sa revalorisation sous le règne de Louis XI. Cette enquête, qui s'arrête à l'aube des guerres de Religion, interroge les logiques du processus d'emblématisation du monument dont la façade harmonique à deux tours est restée associée à la ville de Paris.

\section{Que montre-t-on de Notre-Dame au Moyen Âge?}

Quantitativement, la cathédrale est l'un des monuments les plus représentés de l'imagerie médiévale parisienne. Elle figure dans $20 \%$ des vues de Paris et de sa région que j'ai recensées dans le cadre de ma thèse de doctorat, soit autant que le Palais de la Cité . À la différence du palais généralement représenté pour lui-même, sorti du contexte urbain, et ce dès le XIV ${ }^{\mathrm{e}}$ siècle, la cathédrale apparaît plutôt dans des vues d'ensemble (ou métonymique) de la ville et ce, plus tardivement. Ce sont alors les extérieurs, immédiatement visibles et reconnaissables par les contemporains, qui s'affichent pour la première fois dans les manuscrits du début du $\mathrm{XV}^{\mathrm{e}}$ siècle. Ce sont d'abord les deux tours carrées, terminées en terrasse, que l'on voit émerger au-dessus du tissu urbain à l'horizon, dans le Bréviaire de Châteauroux ou au cœur de l'île de la Cité dans les Heures de Manchester ${ }^{5}$.

\section{Les deux tours occidentales, quel que soit le point de vue sur la ville}

L'adjonction des deux tours de la cathédrale peut suffire à passer d'une image de ville conventionnelle, quelques maisons ceintes d'un mur fortifié, à une image caractérisée de la Ville de Paris. Ainsi en est-il dans l'un des nombreux exemplaires d'À tous nobles, en illustration du récit de la fondation de Lutèce par les Troyens ${ }^{6}$.

3. Isabelle Backouche et al. (dir.), Notre-Dame et l'Hôtel de Ville: incarner Paris du Moyen Âge à nos jours, actes de colloque (21-23 nov. 2013), Paris, Publications de la Sorbonne/Comité d'Histoire de la Ville de Paris, 2016.

4. Raphaële Skupien, «Le peintre et le monument. L'invention du paysage urbain dans la peinture parisienne à la fin du Moyen Âge (XIVe-XVI ${ }^{\mathrm{e}}$ siècles)", dir. É. Hamon, Amiens, Université de Picardie-Jules Verne, 2017, 4 vol. Le catalogue (vol. II) réunit les notices de toutes les images citées ci-après, dans lesquelles sont discutées l'identification des monuments représentés, l'attribution de l'image et sa destination.

5. Châteauroux, Méd. Equinoxe, ms. 2, f. 364 et 367v (Paris, vers 1414); Manchester, The John Rylands University Library, Lat. 164, f. 254 (Paris, vers 1430-1450).

6. New York, Public Library, MA 124, médaillon 30 (Nord de la France, entre 1473 et 1483). 
Dans les vues plus détaillées de la ville, les changements d'orientation de l'église manifestent le caractère distinctif des tours dans la représentation de Notre-Dame. La cathédrale tantôt occidentée dans les Mémoires de Commynes ${ }^{7}$, tantôt tournée vers le nord dans les gravures du début du $\mathrm{XVI}^{\mathrm{e}}$ siècle ${ }^{8}$, se reconnaît invariablement aux deux tours carrées, terminées en terrasse, dont l'une est fréquemment sommée d'une tourelle d'escalier.

Cette représentation de la tourelle d'escalier au sommet de l'une des tours de la cathédrale, nord ou sud selon les peintres, s'inscrit dans une tradition qui met l'accent sur la dissymétrie avérée de la façade. Si dans la réalité les deux tours sont pareillement coiffées d'une tourelle, le clocher nord est plus large que celui du sud ; il abrite une grosse cloche qui a fasciné les hommes du $\mathrm{XV}^{\mathrm{e}}$ siècle :

"Il y a deux clochiers ou il a autant de degrez comme il a de jours en l'an. En l'un est une cloche que l'en puet a peine par quatre fois avironner les bras estendus "?

Les frères Limbourg l'avaient clairement montré avant que Guillebert de Mets ne s'en fasse l'écho. Il n'existe que trois exemples connus de ce type de représentation montrant la structure interne du beffroi nord (ill. 1). Jean Fouquet et le
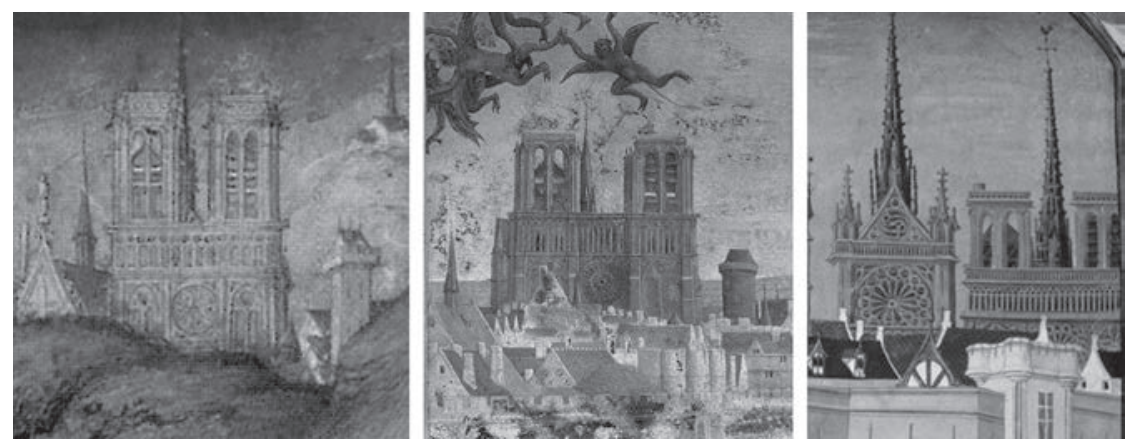

Ill. 1 : Les tours asymétriques de Notre-Dame par les Limbourg, Très Riches Heures de Berry, vers 1411 1416 (@) Chantilly, BAC, Condé 65, f. 51v, détail); Jean Fouquet, Heures d'Etienne Chevalier, vers 1452-1460 (@) New York, The Metropolitan museum of Art, coll. Robert Lehman, inv. 1975.1.2490, détail) ; le Maître du Froissart de Commynes, Chroniques: livre IV de Froissart pour Philippe de Commynes, vers 1470-1472 (@ Londres, BL, Harley 4379, f. 3, détail).

7. Nantes, Musée Dobrée, ms. 18, f. 19 (Paris, entre 1518 et 1524).

8. Cronica cronicarum, Paris : éd. J. Petit et Fr. Regnault, 1521 (impr. J. Ferrebouc, de 1521 à 1541) ; Pierre Gringore, La complainte de la Cité crestienne..., Paris : éd. P. Bige, 1525 (impr. Vve Trepperel, puis J. Ferrebouc, vers 1530); Le registre des ans passez..., Paris : éd. Galliot du Pré, 1533 (impr. A. Cousteau).

9. Guillebert de Mets, La Description de Paris et de l'excellence du royaume de France, cité d'après Antoine Leroux de Lincy et Lazare-Maurice Tisserand, Paris et ses historiens aux XIVe et XVe siècles : documents et écrits originaux recueillis et commentés, Paris, 1867, p. 152-232. Au sujet de l'auteur : Sophie Somers, "The Varied Occupations of a Burgundian Scribe. Corrections and Additions Relating to Guillebert de Mets (c. 1390/1-after 1436) ", dans Bert Cardon, Jan van der Stock et Dominique Vanwijnsberghe (éd.), "Als ich can " - Liber amicorum in Memory of Professor Dr Maurits, Louvain, Peeters, p. 1227-1246. 
Maître du Froissart de Commynes (Philippe de Mazerolles ?) ont repris ce modèle par lequel ils manifestent un intérêt particulier pour leur environnement puisque ce sont les mêmes artistes qui ont, de manière tout à fait exceptionnelle, figuré les parties orientales de l'église.

\section{La ceinture de chapelles et la volée d'arcs-boutants}

Le changement de point de vue sur le monument traduit une certaine familiarité des peintres avec celui-ci. Dans les trois images où se reconnaît le chevet de Notre-Dame, la cathédrale prend le pas sur la ville. Ainsi, à l'arrière-plan de la Descente de croix peinte par Jean Fouquet dans les Heures d'Etienne Chevalier où domine l'imposante silhouette en croix latine de la cathédrale (ill. 2a). Les arcsboutants s'inscrivent en pendant des tours occidentales; conformément au bâti, ils s'insèrent entre les fenêtres hautes vitrées et retombent entre les pignons des chapelles qui émergent au-dessus du tissu urbain.

Le chevet pris dans son carcan d'arcs-boutants a également marqué le Maître du Froissart de Commynes chez qui on en trouve la réminiscence dans des cas de
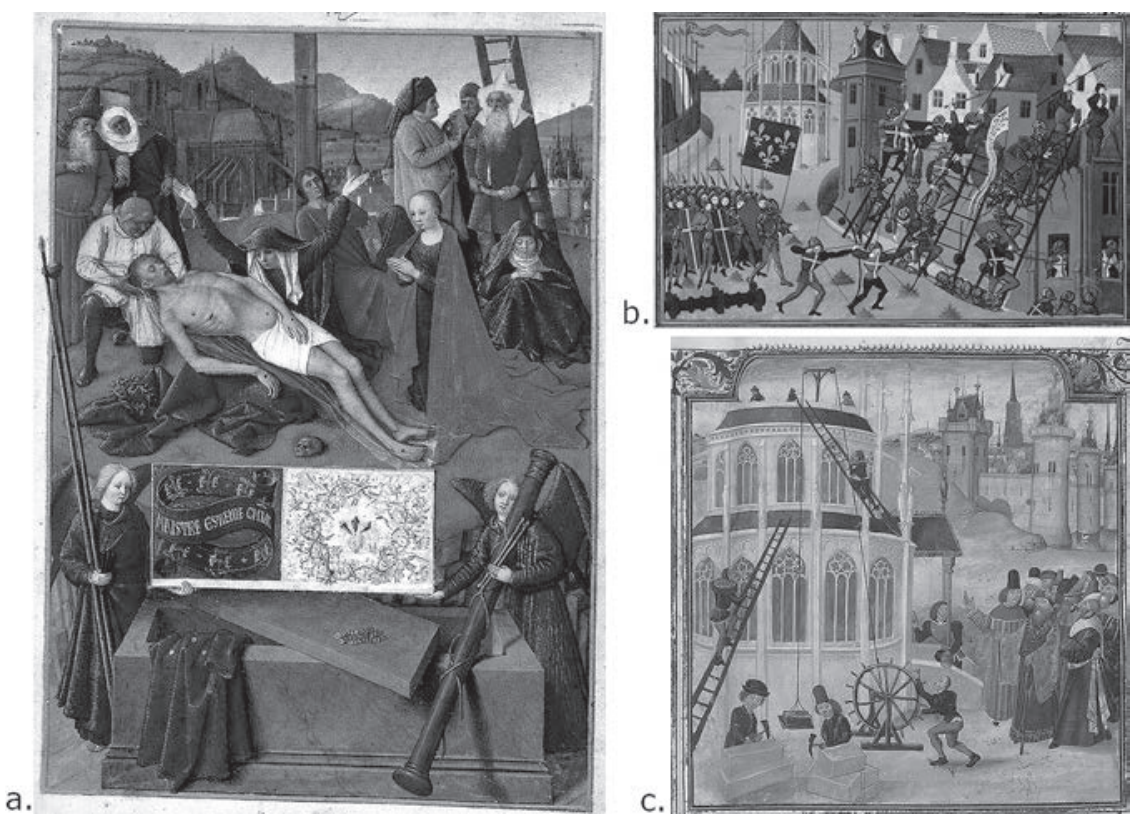

Ill. 2a : Jean Fouquet, «Déploration de la Vierge sur le Christ mort avec, à l'arrière-plan, Paris », Heures d'Etienne Chevalier, vers 1452-1460 (@ Chantilly, BAC, Condé 71) ; b : le Maître du Froissart de Commynes, «Le siège de Pontoise avec Notre-Dame de Pontoise représentée sur le modèle du chevet de Notre-Dame de Paris ", Chroniques du règne de Charles VII de Jean Chartier pour Louis de Bruges, vers 1470-1480 (@ BnF, Fr. 2691, f. 124v) ; c: le Maître du Froissart de Commynes et le Maître de la Chronique d'Angleterre de Vienne, «Le Panthéon de Rome représenté sur le modèle de Notre-Dame de Paris/Pontoise ", Fleur des Histoires de Philippe de Hornes, vers 1480 (@ Copenhague, KB, Thott $5682^{\circ}$, détail du frontispice). 
transposition. Il a utilisé le motif du chevet de Notre-Dame de Paris pour figurer Notre-Dame de Pontoise en marge de la ville assiégée (ill. 2b). Il le reprend encore, en lui donnant une tonalité flamboyante, pour illustrer la fondation de Rome et la construction du Panthéon (ill. 2c). On ne saurait gloser sur la portée symbolique ou politique de ces images : Notre-Dame de Paris appartient à l'imaginaire monumental du peintre.

\section{Vues de détail et méthode de travail}

La fréquentation assidue de l'édifice par les peintres transparaît dans le choix de représenter des détails peu courants. Ces images, particulièrement soignées, sont autant révélatrices de leur connaissance intime du monument que de leurs méthodes de travail. En effet, alors que le portail du Jugement dernier figuré par le maître de Boucicaut reproduit exactement la structure existante et le décor fleurdelysé du soubassement, le Jugement sculpté du tympan est restitué conformément aux types iconographiques picturaux, le Christ sur un arc-en-ciel plutôt qu'en ostentio vulnerum (ill. 3). L'image de la croisée du transept par Jean Fouquet apporte un autre exemple de recomposition fantaisiste. Le peintre, qui a décrit avec une précision extraordinaire la file de piliers coiffés de chapiteaux à crochets, l'a associée à un gâble réminiscent du portail du bras nord (ill. 4). Dans ces deux exemples, l'architecture occupe une place sinon prépondérante du moins équivalente à celle dévolue aux figures. La singularité des thèmes iconographiques suggère en outre que les destinataires des manuscrits, Louis de Guyenne et Étienne Chevalier, étaient en mesure d'identifier le détail représenté. La première figure explicite un rite local
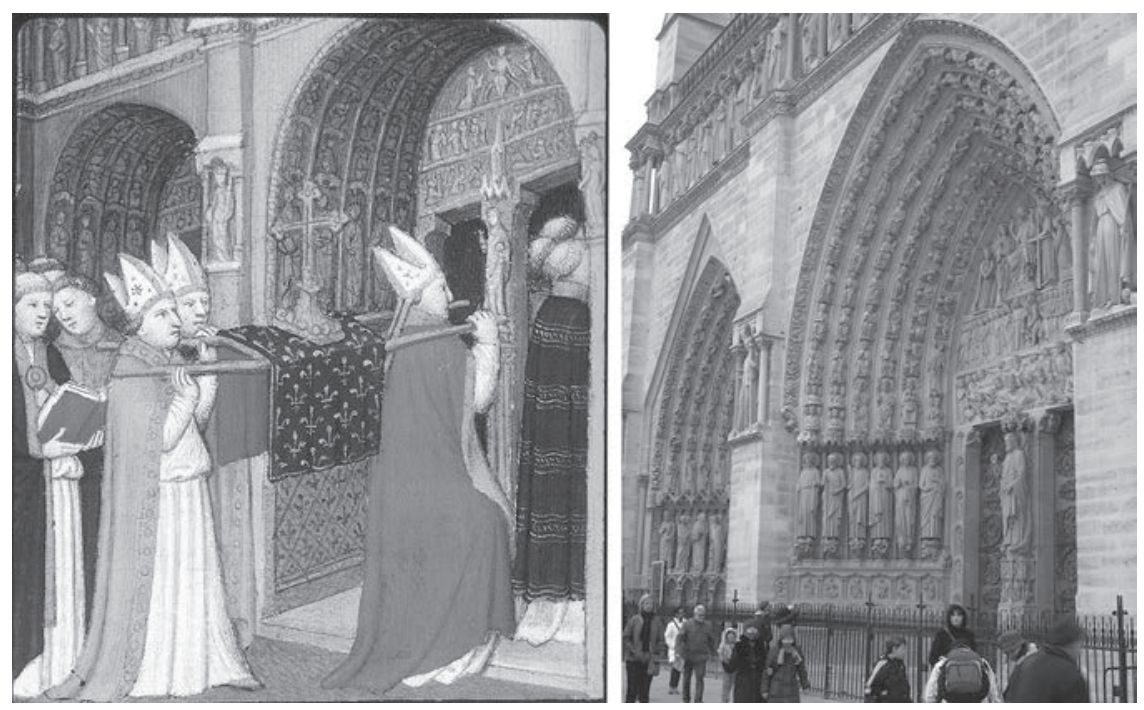

Ill. 3 : Le Maître de Boucicaut, "Le retour de la procession de la croix d'Anseau à Notre-Dame ", Bréviaire de Louis de Guyenne, vers 1414 (ㄷ Châteauroux, Méd. Equinoxe, ms. 2, f. 265) en regard du portail du Jugement dernier (cl. de l'auteur). 

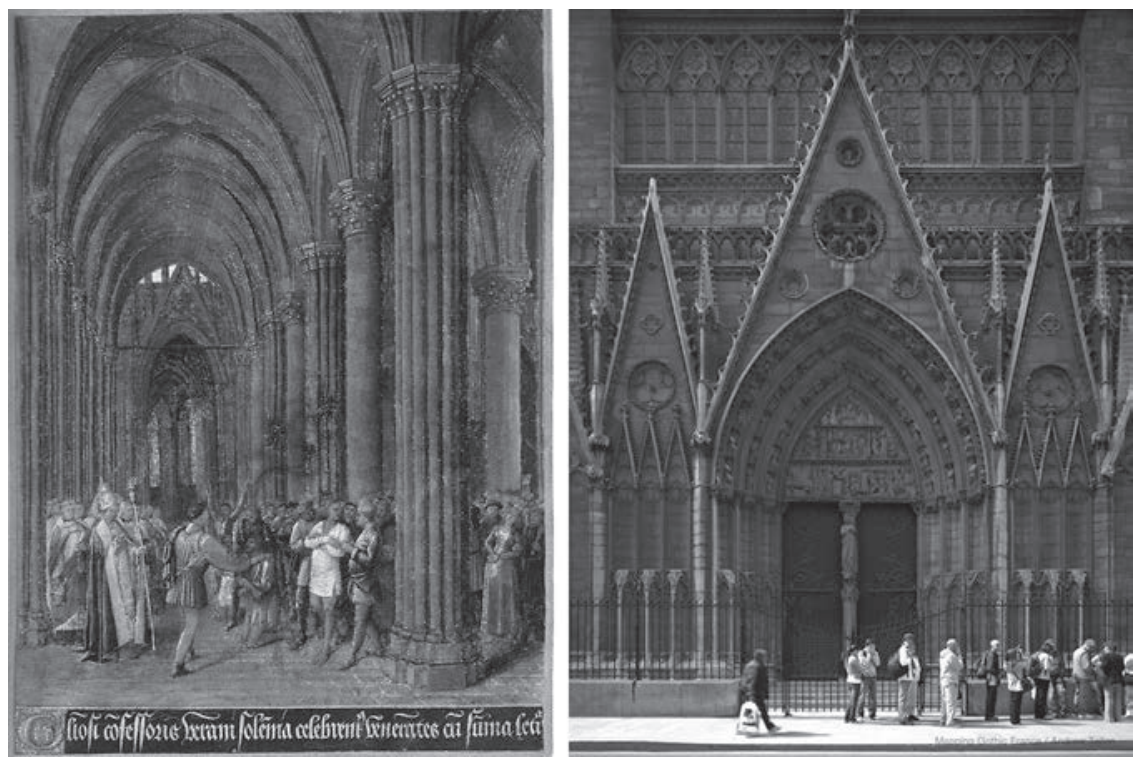

Ill. 4: Jean Fouquet, "Le miracle de saint Vrain transposé dans la nef de Notre-Dame", Heures d'Etienne Chevalier, vers 1452-1460 (C) Paris, musée Marmottan, coll. Wildenstein) en regard du portail du bras nord du transept (cl. Mapping Gothic France / Andrew Tallon).

majeur, la procession des reliques de la Vraie Croix tandis que la seconde évoque un jeu théâtral. Si l'idée du peintre médiéval en peintre de plein air prête à sourire, les sources attestent une pratique du dessin en extérieur dans la préparation de travaux d'utilité publique ou dans le cadre de procédures judiciaires qui nécessitent un travail de mise au net voire de recomposition en atelier ${ }^{10}$. Impliqué dans l'organisation de fêtes civiques, Jean Fouquet a développé sa capacité à projeter un événement dans un environnement monumental familier. Qu'importe alors que le Jugement ne soit pas exactement celui sculpté ou qu'un gable se soit glissé dans le bras du transept?

\section{Les écarts par rapport à la réalité : enjeux esthétiques? symboliques?}

Les descriptions de Notre-Dame, qu'elles soient textuelles ou iconographiques, s'avèrent sélectives. Elles mettent en évidence les éléments les plus caractéristiques du monument aux yeux de leurs auteurs, en fonction de leur degré de familiarité

10. Claude-France Hollard, "Nicolas Dipre cartographe : histoire d'une œuvre ", Revue de l'art, 2010, 167-1, p. 25-30 ; Paul Fermon, Le peintre et la carte. Origines et essor de la vue figurée entre Rhône et Alpes (XIV $-X V^{e}$ siècles), Turnhout, Brepols, 2018 ; Raphäle Skupien, "Représentation du lieu et réception de l'image. La fabrique de la "figure universelle" de la forêt de Thelle d'après le procèsverbal de sa confection (1541) ", Locus, séminaire du LAMOP, organisé par E. Vagnon, C. Fletcher et G. Bührer-Thierry, à Paris, le 15 décembre 2017 (captation audio en ligne). 
avec celui-ci et de l'ampleur de leur culture architecturale. À cela s'ajoutent des modifications majeures apportées par les peintres à l'aspect extérieur de l'édifice, immédiatement perceptibles par leurs contemporains. Les altérations touchent, d'une part, la matérialité de l'architecture ; d'autre part, la partition du frontispice. À l'examen, elles apparaissent moins significatives sur le plan symbolique que révélatrices du goût des contemporains en matière de décor monumental ${ }^{11}$.

\section{La peau de l'édifice: une autre matière, une autre couleur}

Si l'on sait que la cathédrale était peinte, on ne le voit pas dans l'iconographie ni à l'époque médiévale ni à l'époque moderne. Le silence des sources à cet égard est éloquent : mentionner la coloration du décor sculpté est superflu puisque c'est la norme ${ }^{12}$. À lire les chroniqueurs et à se fier aux peintres, on passe à côté des changements de parti de coloration opérés au cours des siècles. Mieux vaut pour cela se reporter aux vestiges archéologiques ${ }^{13}$, éclairés par les relations de voyage des étrangers extra-européens qui, à l'instar de Martyr, ont évoqué les diverses couleurs à dominante de rouge et d'or dont étaient parées les statues de la façade occidentale en 1496 :

"Elle a trois grandes portes tournées du côté du couchant. Les deux battants de la porte du milieu représentent le Christ debout. Au-dessus de cette porte, est le Christ, présidant au jugement dernier. Il est placé sur un trône garni d'or et tout garni d'ornements en or plaqué. [...] Devant le Christ [aux voussures] sont les saints apôtres, les prophètes, les saints patriarches et tous les saints, peints de diverses couleurs et ornés d'or. Cette composition représente le paradis qui enchante le regard des hommes. Au-dessus sont les images des vingt-huit rois, représentés la couronne en tête; ils sont debout sur toute la longueur [de la façade]. Plus haut encore, est la sainte Vierge, mère du Seigneur, ornée d'or et peinte de diverses couleurs " ${ }^{14}$.

11. Raphaële Skupien, "Le Paris du maître du cardinal de Bourbon. Formes et couleurs de l'architecture domestique au XV siècle", dans E. Hamon, M. Béguin et R. Skupien (dir.), Formes de la maison - Du Moyen Âge aux Temps modernes dans le nord de la France, actes de colloque (Amiens, 26-27 mai 2016), textes réunis par M. Quillent, à paraître.

12. En dernier lieu: Denis Verret et Delphine Steyaert (dir.), La couleur et la pierre : polychromie des portails gothiques, actes du colloque (Amiens, 12-14 octobre 2000), Paris, Picard, 2002 ; Anne Vuillemard-Jenn, "La polychromie des façades gothiques et sa place au sein d'un dispositif visuel ", Histoire de l'art, 2013, 72, p. 43-55, que je remercie pour les conseils de lecture donnés.

13. Eugène-Emmanuel Viollet-le-Duc, Dictionnaire raisonné de l'architecture française du XI ${ }^{e}$ au XVI ${ }^{e}$ siècle, Paris, Éd. Bance-Morel, 1854-1868, t. 7, p. 56-109, article "Peinture"; Sylvie Demailly, "L'étude de la polychromie de la façade occidentale de Notre-Dame de Paris ", Monumental. Dossier Notre-Dame, 2000, p. 30-35; Bernard Fonquernie, "Traces de la polychromie sur les portails et la galerie des Rois de Notre-Dame de Paris ", dans D. Verret et D. Steyaert (dir.), La couleur et la pierre..., op. cit., p. 119-128.

14. D’après Martyr, évêque d'Arzendjân (Grande-Arménie), "Mélanges. Statuaire de Notre-Dame de Paris au XV siècle", Annales archéologiques, 1844, t. I, p. 56 (éd. annotée de la traduction de M. Saint-Martin parue dans Journal asiatique, t. IX (1826), p. 323). 
Les peintres ont délibérément écarté ce fait, y compris ceux qui comme le Maître de Saint Gilles étaient réputés pour la fidélité de leurs représentations monumentales ${ }^{15}$. Des enlumineurs moins scrupuleux envers les formes architecturales sont allés jusqu'à changer la couleur de la pierre plutôt que de lui restituer la teinte claire du calcaire ; ils ont opté pour une coloration contrastée avec le tissu urbain alentour. Ainsi, la couleur dorée que lui ont prêtée les illustrateurs de $L a$ Bouquechardière de Jean de Courcy, dans l'exemplaire de Jacques d'Armagnac ${ }^{16}$, et du Pontificale romanum d'Agostino Patrizi Piccolomini, dans une copie partielle pour l'évêque de $\ldots{ }^{17}$, ne parait pas plus significative que la tonalité foncée que lui a donnée Jean Colombe dans le Mamerot de Louis de Laval ${ }^{18}$ : envisager l'usage de l'or sous un angle amélioratif (analogie Paris / Jérusalem céleste) implique d'envisager celui du brun sous un angle péjoratif ce qui est inconcevable de la part du peintre comme de son commanditaire!

Variations sur la position de la galerie des rois:

un effet de la négligence des artistes

Lorsque dans les vues de Paris, Notre-Dame ne tranche pas par sa couleur, elle attire le regard par la rigueur de l'ordonnancement de sa façade qui ne correspond pas à la division de la nef en cinq vaisseaux. La façade se divise en trois travées et trois registres superposés, séparés par deux galeries d'arcature formant deux bandeaux. La première galerie abrite les statues royales, la seconde à claire-voie laisse voir le pignon de l'édifice. Les peintres se sont saisis de cet effet de transparence pour mettre en avant le pignon triangulaire ou, au contraire, le dissimuler derrière les arcatures. Le pignon triangulaire, redoublé par la flèche, accentue l'élan vertical des tours latérales. Ses rampants sont parfois agrémentés de fleurons qui ancrent l'architecture de Notre-Dame dans l'époque gothique.

L'auteur du Plan de Bâle a produit une image originale (ill. 5). Le pignon et les deux tours surplombent le reste du frontispice traité "à l'antique ". La galerie des rois remontée au-dessus de la rose simule une architrave! En accentuant la partition horizontale de la façade, le dessinateur confere un caractère plus antiquisant que gothique à l'architecture de Notre-Dame. C'est une question de style.

\section{Un monument "de style "}

À partir des années 1470, l'imagerie parisienne a débordé le cadre de la production locale. Les peintres flamands puis ligériens s'en sont emparés, probablement à la demande de leurs commanditaires (Louis de Bruges, Louis Mallet de Graville) ou sous l'influence de peintres formés à Paris (entourage du Maître du Froissart de

15. Panneau du retable de Saint Gilles, Washington, National Gallery of Art, Samuel H. Kress coll., Inv. 1952.2.14 (Paris, vers 1500-1505). La couleur blanche bleutée des statues leur donne du relief. C'est une technique picturale proche de la grisaille (utilisée au revers par le peintre) pour rendre le volume de la sculpture plutôt que sa polychromie.

16. La Haye, MMW, 10 A 17, f. 317 (Paris/Carlat, avant 1476).

17. BnF, Lat. 1226 (2), f. 1v (Paris, vers 1530).

18. BnF, Fr. 5594, f. 8v (Les Passages d'outremer de Sébastien Mamerot, Bourges, vers 1474-1475). 


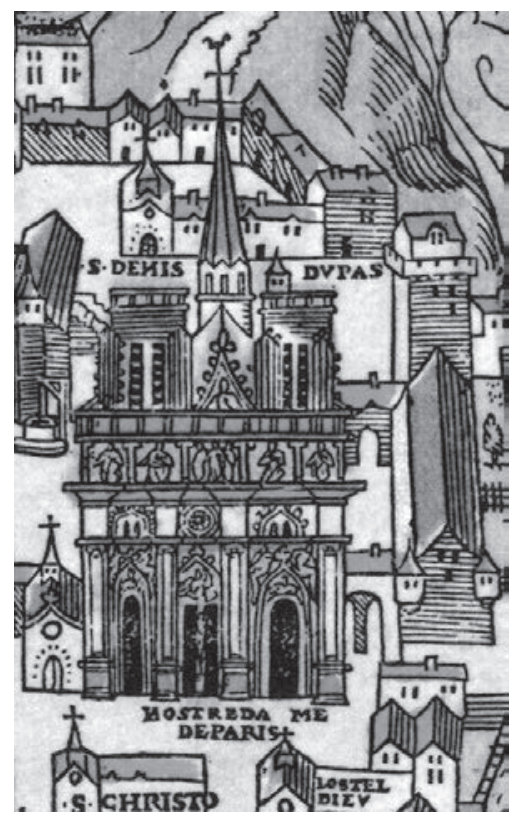

Ill. 5 : Olivier Truschet et Germain Hoyau, Plan de Paris, dit Plan de Bâle, vers 1550 (@) Bâle, Bibliothèque de l'Université).

Commynes à Bruges ou de Jean Fouquet dans le Val-de-Loire). À la différence de leurs homologues parisiens, ils ne connaissent pas directement le monument et n'ont pas la possibilité de se rendre sur place pour vérifier la fidélité de leur représentation. Ils travaillent à partir de modèles qu'ils réinterprètent allègrement. NotreDame de Paris prend ainsi une coloration régionale sous le pinceau de Liévin van Lathem qui, à l'arrière-plan de l'Entrée d'Isabeau de Bavière dans le Froissart de Breslau, a figuré la cathédrale bien plus élancée qu'elle ne l'est en réalité ${ }^{19}$.

D'autres images témoignent, au regard des sources documentaires, de la culture artistique des peintres et de leur volonté de faire ouvre d'art. Romains Buron et André Coulle, actifs sur les chantiers monumentaux de Gisors et de Fontainebleau ${ }^{20}$, ont été chargés de produire la "figure " de la forêt de Thelle dans le cadre d'un procès pendant en Parlement ${ }^{21}$. Leur capacité à reproduire la réalité ne fait aucun

19. Berlin, Staatsbibliothek Preussischer Kulturbesitz, dépôt Breslau I, ms. Rhediger 4, f. 1 (exemplaire d'Antoine de Bourgogne, Bruges, 1468).

20. Etienne Hamon, Un Chantier flamboyant et son rayonnement: Gisors et les églises du Vexin français, Besançon, 2008 ; Marion Seure, "Le prix de la gloire " - Création et commande artistique autour d'une église de la Renaissance: Saint-Gervais-Saint-Protais de Gisors, de 1515 à 1629, thèse dactyl. pour le diplôme d'archiviste paléographe, 2016, 3 vol.

21. Camille Serchuk, "The Polyglot Map : a sixteenth-Century French Forest Map and its Graphic Language(s) ", communication présentée lors du colloque "The Language of Maps" tenu à la Bodleian Library d'Oxford, les 23-25 juin 2011, à paraître ; Raphaële Skupien, "Représentation du lieu... ", op. cit. 


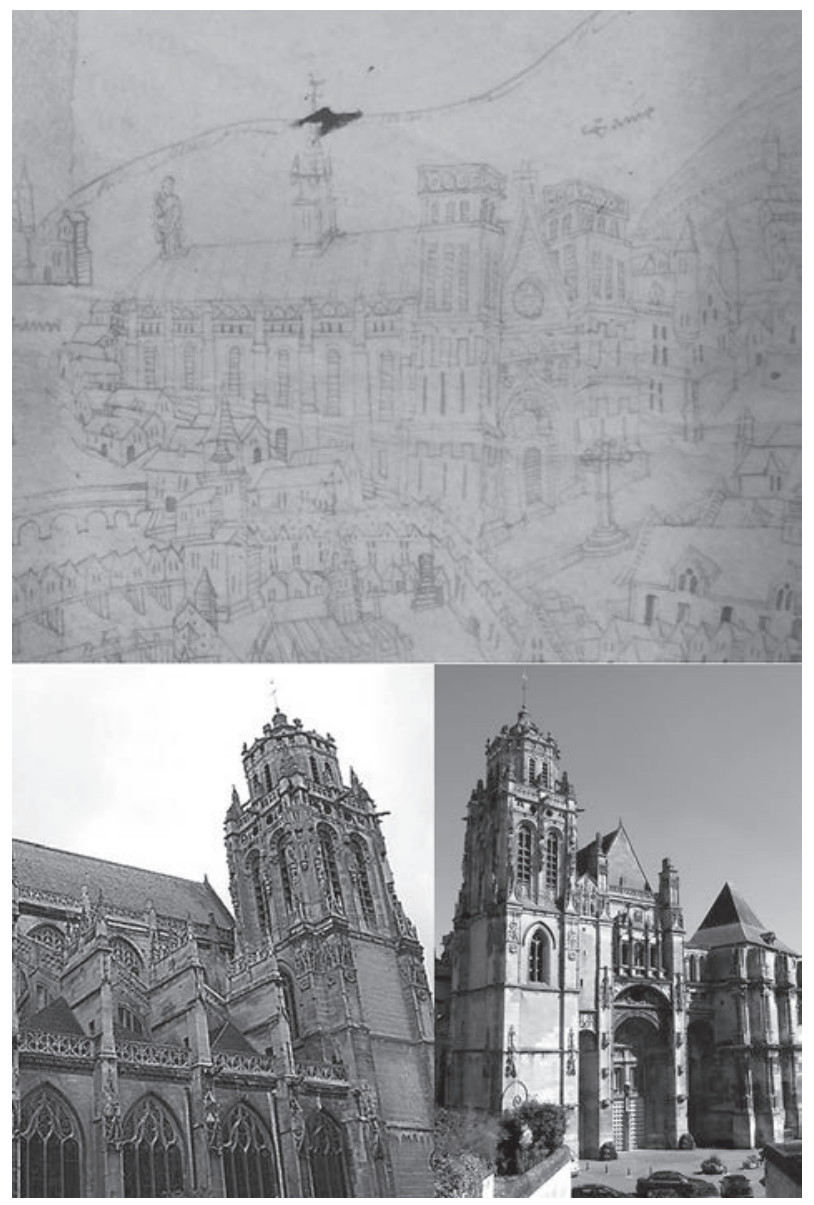

Ill. 6 : La cathédrale de Paris vue par André Buron et Romain Coulle, Figure universelle de la forêt de Thelle - Paris, 1541 (AD Val-d'Oise, 10 J 1500 (3), détail ; cl. de l'auteur) en regard de la collégiale de Gisors (D.R.).

doute pour leurs contemporains puisque cette "figure" a valeur de preuve juridique. Parmi les vues urbaines qui en agrémentent les marges, celle de Gisors avec Saint-Gervais-Saint-Protais confirme l'aptitude des peintres à rendre l'exacte physionomie de l'architecture. La vue de Paris et de sa cathédrale paraît plus fantaisiste (ill. 6) : avec sa nef à deux niveaux d'élévation et son portail unique en plein cintre abrité sous un arc surmonté d'une série d'arcatures couronnées par une frise décorative au-dessus de laquelle s'élève un pignon triangulaire percé d'une petite rose, le tout flanqué de deux tours hors-œuvre surhaussées d'un niveau ajouré, l'édifice évoque la collégiale gisorienne.

Sans pousser le travestissement aussi loin, d'autres peintres ont imprimé à l'édifice leur vision d'une cathédrale plus conforme à leurs idéaux esthétiques ou à ceux 


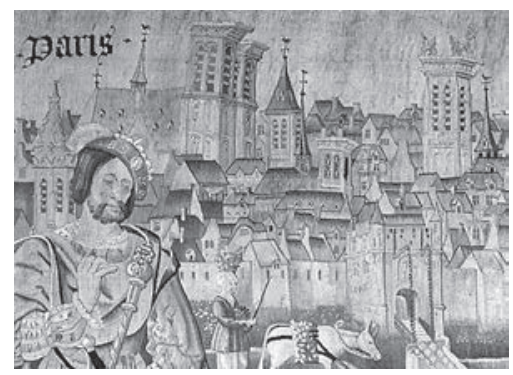

Ill. 7 : Les tours de Notre-Dame et le clocher de Saint-Jacques-de-la-Boucherie d'après un carton de Noël Bellemare et Gauthier de Campes (?), "Paris », Tenture de l'Histoire des Gaules, entre 1522 et 1530 ( Beauvais, musée départemental de l'Oise, 4e pièce, compartiment droit, détail).

de leurs commanditaires. Jean Pichore, dans la scène du convoi funéraire d'Anne de Bretagne, applique à Notre-Dame de Paris son cher parement de marbres polychromes qui recouvre également le baptistère voisin et les maisons alentour ${ }^{22}$. Un souci analogue d'harmonisation du paysage monumental se retrouve dans la Tenture de l'Histoire des Gaules dont l'auteur aura donné un cachet flamboyant aux tours de Notre-Dame, dans le style du clocher de Saint-Jacques-de-la-Boucherie tout juste achevé d'être construit (ill. 7). Ces deux derniers exemples illustrent bien la concurrence des styles dans le milieu parisien autour de 1500 où l'attachement à une tradition flamboyante est une affaire de goût non une méconnaissance de l'art de la Renaissance italienne ${ }^{23}$. À la fin du XV $\mathrm{XV}^{\mathrm{e}}$ siècle, Notre-Dame était devenue le monument incontournable dans les représentations de Paris qui commençaient alors à circuler en Europe.

\section{La valeur emblématique de Notre-Dame de Paris}

La cathédrale fut, avec le palais de la Cité, le monument le plus représenté dans l'imagerie parisienne. Leur histoire paraît cependant bien différente. Le palais l'emporte en ancienneté : il a fait l'objet de plusieurs représentations dès la réfection de la grande salle sous Philippe le Bel. Au contraire de Notre-Dame, le palais est souvent figuré en dehors de son contexte urbain. On apprécie particulièrement les vues intérieures de la grande salle ou du parc du Parlement dues à des peintres locaux. L'image de Notre-Dame a connu un essor tardif, à la faveur de sa reprise par des enlumineurs extérieurs à la ville. On observe dans le même temps un nombre croissant de chantiers d'églises pour lesquels les bâtisseurs s'inspirent explicitement de la cathédrale parisienne. Sa promotion au rang de modèle monumental

22. Paris, Petit Palais-Musée des Beaux-Arts de la Ville de Paris, coll. Dutuit, ms. 665, f. 28 (Le Trépas de l'hermine regretée, Paris, 1514).

23. Mathieu Deldicque, "La commande artistique de l'amiral Louis Malet de Graville (v. 1440-1516) : la recherche d'une autre modernité ", La France et l'Europe autour de 1500, croisements et échanges artistiques, actes de colloque, Paris, Ecole du Louvre, 2015, p. 35-43. 
qui va de pair avec le développement de ses images éclaire sur l'évolution de son statut à l'échelle du royaume.

Un monument pour une ville, pour un royaume:

Notre-Dame de Paris versus le Palais de la Cité

Les plus anciennes vues de la cathédrale sont plus récentes d'un siècle environ à celles du palais de la Cité. Elles apparaissent à partir des années 1410 dans des manuscrits destinés au milieu de la cour, à un dauphin - Louis de Guyenne - et à Jean de Berry, tous deux proches du chanoine Jean Gerson. Jean Gerson a composé le programme iconographique de trois exemplaires du Somnium d'Honoré Bouvet destinés à Jean de Berry, Charles VI et Benoît XIII ${ }^{24}$. L'illustration comprenait une représentation de la Sainte-Chapelle telle qu'elle figure dans le Missel de Guyenne et dans les Très Riches Heures de Berry ${ }^{25}$. Mais, dans les ouvrages dits de librairie, Notre-Dame se signale timidement par ses deux tours carrées noyées dans un panorama urbain bigarré ; elle cède le pas à la Sainte-Chapelle dans les manuscrits du régent anglais ${ }^{26}$.

Au milieu du siècle, Notre-Dame est associée à une certaine idée du royaume et de sa chrétienté comme l'explicite l'auteur d'un guide de lecture du Songe du vieil pèlerin diffusé dans le milieu des officiers royaux après 1452 :

« Pour bien garder le livre. Doctrine des devises et des ystoires [...]

«Le roy et la ville de Paris signifient lui et son royaume qui lui viennent honnourablement au devant, c'est a dire que rendent a Dieu et a son espouse Saincte Mere Eglise une singuliere reverence, amour et obeissance, sans ce que depuis que le noble roy Clovis print le baptesme ayt esté corrompu ne attaint d'aucun blasme de heresie, par quoy il est nommé pardessus tous les roys catholiques le roy tres crestien ${ }^{27}$.

24. Gilbert Ouy, "Une maquette de manuscrit à peintures (Paris, B.N. lat. 14643, Honoré Bouvet, Somnium prioris de Sallono super materia Scismatis, 1394) ", dans Mélanges d'histoire du livre et des bibliothèques offerts à M. Frantz Callot, conservateur en chef de la bibliothèque de l'Arsenal, Paris, 1960, p. 43-51.

25. Resp. Paris, Bibl. Mazarine, ms. 406, f. 7 (Paris, vers 1415) ; Chantilly, BAC, Condé 65, f. 158 (Paris, vers 1411-1416; Bourges, vers 1485).

26. BnF, Lat. 17294, f. 243 (Bréviaire de Bedford, Paris, vers 1424-1435).

27. Cité d'après Philippe Contamine, "Un préambule explicatif inédit dans un manuscrit (milieu du $\mathrm{XV}^{\mathrm{e}}$ siècle) du Songe du vieil pèlerin (1389) de Philippe de Mézières : le texte et l'image ", CRAI, 2007, 151 ${ }^{\mathrm{e}}$ année, no 4, p. 1901-1923; à croiser avec Alessia Marchiori, "Forgier fins besans » - Le Songe du Vieil Pelerin de Philippe de Mézières : projet sotériologique et pouvoir de l'écriture à la fin $d u X I V^{e}$ siècle, thèse de doctorat dactyl., dir. A. M. Babbi et J. Cerquiglini-Touvet, Universités de Vérone et de Paris-Sorbonne, 2014, qui s'est intéressée au travail de réécriture du texte sous Charles VII. 


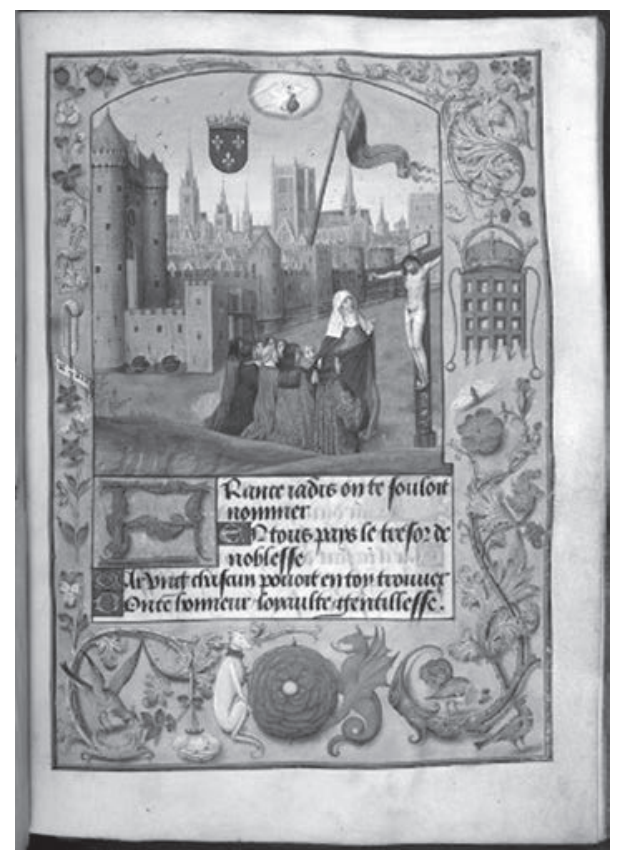

Ill. 8 : Scène d'intercession devant Paris, en illustration de la ballade de Charles d'Orléans «France, jadis on te souloit nommer...", pour Edouard IV, mort en 1483 (C) BL, Royal MS. 16 F II, f. 89).

Alors que l'aire italienne est longtemps restée hermétique aux représentations de Paris et de ses monuments ${ }^{28}$, les milieux de la cour de France dans le Val-deLoire, de la cour de Bourgogne à Bruges, puis de la cour d'Angleterre à Londres les ont volontiers reçues ${ }^{29}$. Une vue de Paris avec la porte Saint-Denis et les tours de Notre-Dame illustre ainsi le poème France, jadis... de Charles d'Orléans dans un manuscrit réalisé à Bruges pour Edouard IV (ill. 8). Cette image de la cathédrale de Paris s'inscrit en pendant d'une représentation de la tour de Londres. Pour les étrangers, il semble admis que le monument parisien par excellence, celui que l'on repère à distance dans le paysage, est la cathédrale.

\section{Un modèle monumental controversé}

Le clergé du diocèse a, dès le $\mathrm{XII}^{\mathrm{e}}$ siècle, choisi de rénover ses églises sur le modèle de la cathédrale de Maurice de Sully, sans doute pour affermir sa filiation

28. Dans son introduction aux Plans de Paris, des origines (1493) à la fin du XVIII siècle : étude, cartobibliographie et catalogue collectif, Paris, BnF, 2002, Jean Boutier s'appuie sur une lettre du marquis de Gonzague pour expliquer qu'il n'existe pas de vue reconnaissable de Paris avant le $\mathrm{XVI}^{\mathrm{e}}$ siècle en dépit de l'entreprise d'H. Schedel (1493).

29. Notons que toute représentation d'une église à deux tours carrées ne saurait être identifiée avec une image de Notre-Dame de Paris, surtout dans le milieu des enlumineurs flamands familiers autant des vues urbaines que de ce type d'église comme l'attestent les portraits précoces de Sainte-Gudule de Bruxelles. 
avec l'église épiscopale ${ }^{30}$. Mais, à l'exception de la collégiale de Mantes dont la façade copie celle de Notre-Dame de Paris, les autres églises citent ponctuellement son plan ou son élévation intérieure. Il faudra aller plus loin (Meaux), avancer dans le temps (Abbeville) pour prendre la mesure de son rayonnement ${ }^{31}$. Le caractère novateur du chantier parisien se révélait aux yeux des contemporains au fil de l'avancement des travaux. Il s'ensuivit une période de creux relatif avant que NotreDame ne suscite un regain d'intérêt comme à Troyes où le clergé, hésitant sur le parti à suivre pour l'achèvement de la façade, a mandé le peintre Pierre Trubert pour lever le portrait des cathédrales d'Amiens, de Reims et de Paris en 1453.

$\mathrm{Au} \mathrm{XV}$ siècle, tout le monde n'est pas subjugué par Notre-Dame de Paris. Les textes littéraires en témoignent ${ }^{32}$. La fabrique de Milan, qui a fait venir un peintre et un architecte de Paris pour les assister dans la construction du Dôme, redoute cependant que les solutions proposées par ces derniers n'aboutissent à la mise en œuvre d'un édifice aussi sombre que Notre-Dame, beaucoup trop sombre ${ }^{33}$.

Les contemporains du chantier ont sans doute été aussi étonnés que Viollet-leDuc par l'abandon des flèches de couronnement. C'est pourtant dans le parti du couronnement des tours en terrasse que l'édifice a de nouveau trouvé grâce aux yeux des bâtisseurs autour de $1500^{34}$. Ce parti, évocateur d'un art de bâtir carolingien voire antique, s'accorde avec les discours sur les origines mythiques de la cathédrale réputée avoir été fondée par Charlemagne. En vertu de leur ancienneté réelle ou supposée, les tours de Notre-Dame de Paris ont contribué à faire de la cathédrale un monument historique, "à la mode " plutôt qu' «à la moderne ".

Dans son Discours des cérémonies sur le mariage d'Anne de Foix (1512), l'humaniste Pierre Choque, à qui nous laissons le dernier mot, campe le récit des origines troyennes du royaume de France dans un cadre urbain clairement inspiré par Paris. Le secrétaire d'Anne de Bretagne situe ainsi dans la ville de Bude une grande église Notre-Dame fondée par Charlemagne... un de ces monuments qui donne «apparence que autreffoys y a eu de grans edifices » à Bude/Paris :

«Pour revenir à la beauté de la situation desdictz ville et chasteau de Bude ou y a plusieurs Turcqs prisonniers, aussy y a il par tout le Royaume, es

30. Mathieu Lheure, Le Rayonnement de Notre-Dame de Paris dans ses paroisses (1170-1300), Paris, Picard, 2010 ; Dany Sandron, "Notre-Dame, une architecture diocésaine et royale » dans Julerot et al., 2013, p. 29-35.

31. Dany Sandron dans Notre-Dame, op. cit., 2012, p. 92-93, rappelle le cas étudié par Peter Kurmann de la cathédrale de Meaux reconstruite au XIII ${ }^{e}$ siècle selon un plan à cinq vaisseaux précédés d'une façade harmonique à trois portails. On pourrait aussi citer le plan de la cathédrale de Bourges, les galeries des rois des cathédrales de Chartres, d'Amiens et de Reims, les pignons du transept de la cathédrale de Rouen, la façade de la collégiale d'Abbeville... et, à l'étranger, le chevet de la cathédrale de Bâle.

32. On lira à ce sujet la contribution de Judith Förstel, dans ce même numéro.

33. Annali della fabrica, p. 197-198.

34. Étienne Hamon, Une Capitale flamboyante - La création monumentale à Paris autour de 1500, Paris, Picard, 2011. 
environs, plusieurs ebatemens et passe temps de prince, comme de l'isle de Chepelle appartenant a ladicte dame a cause de son douaire, situee en une lieue de ladicte ville ou meillieu dudict fleuve, en laquelle y a maison et jardins de bonne sorte ; l'isle est de longueur de quinze ou seize lieues, bien fertille, multipliee de grant nombre de bestes rousses, comme de cerfs, biches et lievres. Et viz ung jour que ladicte dame estoit en icelle isle, au bruit et marcher des chevaulx lever quinze ou seize lievres en moins de pays que troys traictz d'arc. Au dessus de ladicte ville, amont la riviere a une place de Nostre Dame, laquelle dit on estre la premiere eglise que fonda jamais l'empereur Charlemaigne, ainsy que appart par une espitaphe y estant. $\mathrm{Au}$ plus pres est Bude Veige ou y a beau lieu et grant nombre d'esglises et maisons. Et en icelluy lieu fut premierement ediffié Bude ; et est celle Bude Veige situee sur le bort du fleuve et ou cyrcuit de la ville de Sicambrie ou habiterent premierement les Françoys lors nommez Sicambriens, quant Troye fut destruicte et mise en exil. Et si a apparence que autreffoys y a eu grans edifices... ${ }^{35}$.

Raphaële SKUPIEN

Docteure en Histoire de l'art

Chargée de cours à l'Université d'Amiens et de Lille Chercheure associée de TrAme (E.A. 4284)

Membre de l'Association des scientifiques pour la restauration de Notre-Dame

35. D’après Discours des cérémonies du mariage d'Anne de Foix, éd. Antoine Leroux de Lincy, 1861, p. 437-438. 
